\title{
Thermal measurements of stationary nonequilibrium systems: A test for generalized thermostatistics
}

\author{
Damián H. Zanette \\ Consejo Nacional de Investigaciones Científicas y Técnicas, Centro Atómico \\ Bariloche and Instituto Balseiro, 8400 San Carlos de Bariloche, Río Negro, \\ Argentina \\ Marcelo A. Montemurro \\ Abdus Salam International Centre for Theoretical Physics, Strada Costiera 11, \\ 34100 Trieste, Italy
}

\begin{abstract}
We show that a gas thermometer in contact with a stationary classical system out of thermal (Boltzmann) equilibrium evolves, under very general conditions, towards a state characterized by a Lévy velocity distribution. Our approach is based on a kinetic-like equation that applies to a wide class of models for the systemthermometer interaction. The results clarify the role of non-exponential energy distributions as possible generalizations of the Boltzmann distribution for systems where the usual formulation of thermostatistics may not apply. In particular, they show that the power-law distributions derived from Tsallis's nonextensive formalism are irrelevant to the stationary state of the thermometer, thus failing to give a consistent description of the system-thermometer equilibrium. We point out the need of a generalized thermostatistical formulation able to give a unified frame to Lévy and Maxwell distributions.
\end{abstract}

Key words:

Non-equilibrium systems, Kinetic theory, Lévy distributions, Generalized thermostatistics

PACS: 05.20.-y, 05.70.-a, 05.20.Dd

Email addresses: zanette@cab.cnea.gov.ar (Damián H. Zanette), mmontemu@ictp.trieste.it (Marcelo A. Montemurro). 
Lévy distributions [1] have been extensively applied to the description and modeling of a wide class of physical processes, ranging from anomalous transport in disordered media [2] and turbulent flows [3], to phase-space diffusion in dynamical systems [4] and polymer dynamics [5]. They have also found application in other branches of science, such as in biology [6,7]. The ubiquity of Lévy distributions in many natural phenomena is a straightforward consequence of their stable character under summation of random variables whose distributions have diverging moments, as shown by Paul Lévy in his generalization of the central limit theorem [8]. This essential result gives Lévy distributions the same status as the Gaussian distribution in the statistical description of stochastic processes.

While most applications of Lévy distributions deal with dynamical and transport processes, we show in this Letter that they replace the equilibrium Maxwell distribution - a Gaussian in the velocity variable - in an extended version of the scenario of a thermal measurement. We analyze the asymptotic energy distribution of a thermometer in thermal contact with a classical system in a nonequilibrium stationary state. While the nature of this nonequilibrium state is not explicitly specified, we typically refer to a situation where the system is not isolated and suffers the effect of external stationary forces that maintain its energy distribution apart from the prediction of Boltzmann-Gibbs statistics. It is an essential fact of thermostatistics [9] that, if the system is in thermodynamical equilibrium, its Boltzmann energy distribution is "copied" by the thermometer in such a way that its average energy per degree of freedom, i.e. its temperature, becomes equal to that of the system. Now, what properties of the state of the system are detected by the thermometer in the case that the system is maintained out of equilibrium?

The motivation of this question is two-fold. First, since all physical systems are to some extent thermodynamically open and subject to external influence, the possible effect of these factors in a thermal measurement constitutes a problem of empirical relevance. In general, this problem transcends the limits of equilibrium thermostatistics and calls for a nonequilibrium, dynamical formulation. We choose a kinetic-like approach which describes a wide class of interaction models for the thermal contact between the system and a gas thermometer. Our results show that the thermometer attains a velocity distribution which is fully determined by the high-energy distribution of the system, and results to be insensitive to other details in the system state. In the case where the energy distribution of the system decays as a power law, as advanced above, the thermometer velocities approach a Lévy distribution. This distribution "copies" the singularities associated with divergent energy moments in the system, and reduces to a Maxwellian when the average energy is finite.

The second motivation for our question has to do with the possibility of gen- 
eralizing thermostatistics to the description of physical systems where the usual Boltzmann-Gibbs formulation may not apply [10]. Tsallis's nonextensive thermostatistics, for instance, is claimed to replace the usual formulation for systems where long-range interactions lead extensivity assumptions to break down, while containing Boltzmann-Gibbs statistics as a limiting case [11]. In Tsallis's formalism, the Boltzmann exponential distribution is replaced by a class of power-law functions derived from a variational principle for a generalized entropy. Whether this formalism is compatible with the basic facts of equilibrium between systems in thermal contact can be decided, precisely, by studying the interaction of a thermometer and a system with such power-law energy distribution. Our results show that, since Lévy distributions are not regarded in this formalism as possible equilibrium states, Tsallis's thermostatistics fails to give a consistent picture of thermodynamical equilibrium.

Consider a system in a stationary state which does not necessarily coincide with Boltzmann equilibrium. We assume that the state of the system is macroscopically characterized by an energy distribution $F_{0}(\epsilon)$. The system interacts with a thermometer consisting of an ensemble of independent particles of mass $m$ moving in a $d$-dimensional domain. Within this domain, the distribution of particles is supposed to be spatially homogeneous and isotropic in velocities, such that the associated distribution function $F(\epsilon, t)$ depends on the energy $\epsilon$ and the time $t$ only. The interaction between system and thermometer is assumed to fulfill the following conditions. (i) Interaction events are time localized and infrequent, such that the typical time between interactions is large as compared with the relaxation time of the energy distribution of the system toward $F_{0}(\epsilon)$. This insures that (a) at each event, the system is found at its stationary state and (b) any correlation between the states of system and thermometer, created by the interaction, dies out before the next event takes place. (ii) The amount of energy interchanged at each event is small as compared with the total energy of both the system and the thermometer.

Within these conditions, it is possible to describe the evolution of $F(\epsilon, t)$ by means of a kinetic-like equation. On the other hand, as a consequence of condition (i), no evolution equation is required for the system distribution. To represent the interaction between system and thermometer we choose a very generic class of models - closely related to stochastic Maxwell models [12] which considerably simplifies the mathematical treatment of the kinetic problem and, at the same time, does not imply severe limitations to the physics of the interaction. In fact, these models include interactions where momentum conservation can either hold or be violated, and admit arbitrary angular dependence in the associated cross sections. They assume that the interaction rate is independent of the energy and that energy itself is conserved. The corresponding evolution equation for $F(\epsilon, t)$ stands for the balance between events where, due to interaction with the system, the thermometer reaches or abandons its states of energy $\epsilon$. Such events yield, respectively, positive and 
negative contributions to $\partial_{t} F$. The equation is more conveniently written in the Fourier representation, for the Fourier-transformed distribution

$$
\Phi(u, t) \equiv \phi(k, t)=\int \exp (-i \mathbf{k} \cdot \mathbf{v}) f(v, t) d v^{d}
$$

with $u=k^{2} / 2 m$. Here,

$$
f(v, t)=\frac{\Gamma(d / 2)}{2 \pi^{d / 2}} m v^{2-d} F\left(m v^{2} / 2, t\right)
$$

is the (isotropic) velocity distribution associated with $F(\epsilon, t)[12]$. Due to the normalization of $F(\epsilon)$, we have $\Phi(0, t)=1$ for all $t$. With an analogous definition for $\Phi_{0}(u)$ in terms of $F_{0}(\epsilon)$, the Fourier-transformed evolution equation reads $[12]$ :

$$
\partial_{t} \Phi(u, t)=\int_{0}^{1} w(s) \Phi_{0}(s u) \Phi[(1-s) u, t] d s-\Phi(u, t) .
$$

The kernel $w(s)$ characterizes the interaction, and satisfies the normalization $\int_{0}^{1} w(s) d s=1$. When necessary, we assume that the moments

$$
w_{\mu}=\int_{0}^{1} s^{\mu} w(s) d s
$$

are well defined.

The above condition (ii) imposes that $w(s)$ is appreciably different from zero only for $s \approx 0$. Taking this condition into account, we write $\Phi[(1-s) u, t] \approx$ $\Phi(u, t)-s u \partial_{u} \Phi(u, t)$, to obtain an approximate form of Eq. (3). Its solution can be shown to asymptotically approach the stationary distribution

$$
\Phi(u)=\exp \left[\frac{1}{w_{1}} \int_{0}^{u} \frac{d u^{\prime}}{u^{\prime}} \int_{0}^{1} w(s) \ln \Phi_{0}\left(s u^{\prime}\right) d s\right] .
$$

Since $w(s) \approx 0$ except for $s \approx 0$ the asymptotic solution $\Phi(u)$ is fully determined by the behavior of $\Phi_{0}(u)$ close to $u=0$. We pay particular attention to the special case where, for $u \approx 0$,

$$
\Phi_{0}(u) \approx 1-\alpha_{0} u^{\nu}
$$

with $\alpha_{0}>0$ and $0<\nu \leq 1$. For $\nu \neq 1$, this form corresponds to an energy distribution with a power-law tail for high energies, $F_{0}(\epsilon) \sim \epsilon^{-\nu-1}$. While $F_{0}$ 
is still normalized, its first moment - namely, the average energy - diverges. In the limit $\nu=1$ a finite average energy is recovered and the long high-energy tail is lost. For $\Phi_{0}(u)$ as in Eq. (6), the stationary Fourier distribution for the thermometer is

$$
\Phi(u)=\exp \left(-\alpha u^{\nu}\right)
$$

with $\alpha=\alpha_{0} w_{\nu} / \nu w_{1}$. Note that, for $0<\nu<1, \Phi(u)$ reproduces the singularity of $\Phi_{0}(u)$ at $u=0$. The corresponding Fourier-transformed velocity distribution, $\phi(k)=\Phi\left(k^{2} / 2 m\right)$ [see Eq. (1)], reads

$$
\phi(k)=\exp \left(-b k^{\gamma}\right)
$$

with $b=\alpha /(2 m)^{\nu}$ and $\gamma=2 \nu(0<\gamma \leq 2)$. Note that, in the velocity representation, this corresponds to a Lévy distribution for each velocity component. In fact, the Fourier transform of, say, $f\left(v_{x}\right)=\int d v_{y} d v_{z} \ldots f(v)$ is $\phi\left(k_{x}, 0,0, \ldots\right)=\exp \left(-b\left|k_{x}\right|^{\gamma}\right)$. Though, in general, it is not possible to write explicitly the corresponding form of $f\left(v_{x}\right)$, it is known that for large $\left|v_{x}\right|$ and $\gamma \neq 2$ it behaves as $f\left(v_{x}\right) \sim\left|v_{x}\right|^{-\gamma-1}$ [1]. For $\gamma=2, f\left(v_{x}\right)$ is a Gaussian distribution.

We stress that the result (8) for the Fourier-transformed velocity distribution of the thermometer is independent of the detailed form of the distribution $F_{0}(\epsilon)$ of the system at low energies. As long as condition (ii) is satisfied, $\phi(k)$ is completely determined by the power-law high-energy tail of $F_{0}(\epsilon)$.

Let us analyze these results in the few cases where the velocity distribution can be explicitly obtained. We first consider the situation where the average energy of the system under study is finite, $E=\int \epsilon F_{0}(\epsilon) d \epsilon<\infty$. Under this condition we have, in Eq. (6), $\alpha_{0}=2 E / d$ and $\nu=1$. This gives, for the Fourier-transformed velocity distribution of the thermometer, the Gaussian $\phi(k)=\exp \left(-E k^{2} / m d\right)$. In the velocity representation we get the Maxwellian

$$
f(v)=\left(\frac{m d}{4 \pi E}\right)^{d / 2} \exp \left(-\frac{m d}{4 E} v^{2}\right)
$$

The average value of the kinetic energy over this distribution is, as may be expected, $\left\langle m v^{2} / 2\right\rangle=E$. In other words, the stationary velocity distribution of the thermometer is a Maxwellian whose average energy per particle coincides with that of the system. This result includes of course the case where the system is itself in thermodynamical (Boltzmann) equilibrium at temperature $T$, for which $E \propto T$.

For $\nu=1 / 2$, the energy distribution of the system decays as $F_{0}(\epsilon) \sim \epsilon^{-3 / 2}$ and, 
consequently, the average energy diverges. The Fourier-transformed velocity distribution of the thermometer results to be $\phi(k)=\exp (-b k)$, with $b=$ $\alpha_{0} w_{1 / 2} \sqrt{2 / m w_{1}^{2}}$. Taking into account its units, we write $b=p_{0} / m$, where $p_{0}$ is a characteristic linear momentum given by the energy distribution $F_{0}(\epsilon)$ and the specific form of the interaction kernel $w(s)$. In velocity space, each component $v_{i}$ is distributed according to a Cauchy distribution,

$$
f\left(v_{i}\right)=\frac{m p_{0}}{\pi} \frac{1}{p_{0}^{2}+m^{2} v_{i}^{2}}
$$

Note that, here, the relevant dynamical variables in the thermometer distribution are the momentum components $m v_{i}$ and, accordingly, the relevant parameter is the momentum $p_{0}$. Compare with the result for $\nu=1$, Eq. (9), where the distribution is a function of the energy and the relevant parameter is $E$.

The present results shed light on the role of non-Boltzmannian energy distributions in defining the stationary states of out-of-equilibrium interacting systems. In particular, they show that, under the above conditions (i) and (ii), the Gaussian stationary velocity distribution of an ensemble of independent particles interacting with a non-equilibrium stationary system results to be a special instance of the more general situation where the velocity components have Lévy distributions. We stress that, despite the fact this conclusion is limited by the validity of the assumptions (i) and (ii), these conditions are fully compatible with the paradigm of thermal measurement [9] and therefore refer to a realistic, experimentally accessible situation.

As advanced above, our conclusions are particularly significant in the evaluation of generalized formulations of thermostatistics as plausible descriptions of physical systems to which the usual Boltzmann-Gibbs theory may not apply. We focus our attention on Tsallis's formulation, which has motivated large amounts of work over the last two decades [10]. Within Tsallis's statistics, canonical probability distributions are obtained from maximization of a generalized entropy [11]

$$
S_{q}=-\frac{1-\sum_{i} p_{i}^{q}}{1-q},
$$

where $p_{i}$ is the probability of state $i$, and the sum runs over all the states of the system. The parameter $q$ quantifies the nonadditivity of $S_{q}$, and is therefore a measure of nonextensivity in the system under consideration. The maximization process is subject to the generalized constraint [13]

$$
E_{q}=\frac{\sum_{i} \epsilon_{i} p_{i}^{q}}{\sum_{i} p_{i}^{q}}
$$


with $\epsilon_{i}$ the energy of state $i$, and leads to the probability distribution

$$
p_{i} \equiv p\left(\epsilon_{i}\right)=Z_{q}^{-1}\left[1-(1-q) \beta_{q}\left(\epsilon_{i}-E_{q}\right)\right]^{1 /(1-q)},
$$

where $\beta_{q}$ is a ("renormalized" [13]) Lagrange multiplier, analogous to the inverse temperature, and $Z_{q}$ is a normalization constant, analogous to the partition function. Boltzmann-Gibbs formulation is recovered for $q \rightarrow 1$. Note that, for systems whose density of states behave as $\rho(\epsilon) \propto \epsilon^{\sigma}$-a class which includes most classical systems - the probability $p(\epsilon)$ corresponds to a Fouriertransformed energy distribution of the form of Eq. (6), with $\nu=-\sigma+(q-$ $2) /(1-q)$.

An attractive feature of Tsallis's generalized thermostatistics is that it preserves most of the mathematical structure of the usual theory, including the definition of thermodynamic functions, Legendre transformations, and even linear nonequilibrium properties [10]. In fact, a large part of the literature on this topic is devoted to the formal extension of thermodynamical relations to the generalized formulation. Thus, putting aside its phenomenological applications to nonequilibrium processes such as anomalous diffusion [14] and turbulence [15], the real tour de force of Tsallis's formalism lies in the description of thermal equilibrium for systems where Boltzmann-Gibbs theory is supposed to fail. This is in fact the original and most frequently invoked motivation of the formulation and, consequently, constitutes its genuine source of validation. Our results clarify whether Tsallis's statistics is relevant to an aspect that - in spite of its essential role in thermodynamics - has been scarcely treated in the profuse literature on Tsallis's theory [16], namely, thermal equilibrium between interacting systems.

Assume to have a system which, due to its nonextensive nature, exhibits an energy distribution of the form of Eq. (13), as predicted by Tsallis's thermostatistics. Allow furthermore the system to interact with a thermometer, as specified above. Consider first that the nonextensivity index $q$ and the density of states of the system are such that $E=\sum_{i} \epsilon_{i} p_{i}<\infty$. According to our results, the stationary velocity distribution of the thermometer is a Maxwellian, corresponding to a Boltzmann exponential probability for the energy. As expected for an ensemble of independent particles, the thermometer behaves as an extensive system $(q=1)$. The value of the parameters in the thermometer distribution predicted by Tsallis's formalism, however, results to be wrong. Abe and Rajagopal [16] have shown that a formal extension of equilibrium conditions for two systems in thermal contact yields, in Tsallis's theory, $\beta_{q}=\beta_{q^{\prime}}$, where $q$ and $q^{\prime}$ are the nonextensivity indices corresponding to the two systems [17]. Our results show that this relation is generally not satisfied. In fact, taking $q^{\prime}=1$ as the nonextensivity index of the thermometer, Eq. (9) implies $\beta_{q^{\prime}} \propto E^{-1}$. On the other hand, $\beta_{q}$ shows in general a more complicated dependence on $E$. For instance, it can be readily shown that, for a system with 
a density of states $\rho(\epsilon) \propto \epsilon^{\sigma}$, one has $\beta_{q} \propto E^{(q-1)(\sigma+1)-1}$. Let us also point out that the generalized average $E_{q}$ of Eq. (12) plays no role in the parameter that defines the equilibrium distribution of the thermometer-namely, the inverse temperature $\beta_{q^{\prime}}$-in spite of the fact that this generalized average replaces $E$ in the formal extension of thermodynamics to Tsallis's formalism.

In the case that the average $\sum_{i} \epsilon_{i} p_{i}$ diverges, our results imply that the thermometer approaches an energy distribution not belonging to the class of the system distribution, Eq. (13). Thus, no well-defined index $q$ can be assigned to the thermometer. In this situation, the assumption that Tsallis's thermostatistics would describe the system-thermometer equilibrium fails drastically. The only trace of the energy distribution of the system in the thermometer distribution arises from its power-law tail, which defines the Lévy exponent $\gamma=2 \nu$ in Eq. (8). Note that, again, the generalized average $E_{q}$ is irrelevant to the determination of the equilibrium distribution of the thermometer.

Summing up, our kinetic description of a thermometer in contact with a system in a stationary state different from Boltzmann equilibrium, suggests that a suitable extension of thermostatistics should yield Lévy distributions as the generalization of the Maxwellian velocity distribution, instead of the powerlike functions of Eq. (13). We conclude that the energy probabilities derived

from Tsallis's thermostatistics do not play the role of equilibrium distributions for systems in thermal contact. Though this conclusion is not completely general, it applies to a wide class of interaction models in the realistic situation where a system is put in contact with a thermometer. The question remains open as to which form of the entropy and which constraints should be used to derive Lévy distributions from a variational principle.

We thank G. Abramson and I. Samengo for their critical reading of the manuscript.

\section{References}

[1] E. W. Montroll and B. J. West, in Studies in Statistical Mechanics: Fluctuation Phenomena, E. W. Montroll and J. L. Lebowitz, eds. (North Holland, Amsterdam, 1979).

[2] J. P. Bouchaud and A. Georges, Phys. Rep. 195, 127 (1990).

[3] E. R. Weeks, T. H. Solomon, J. S. Urbach and H. L. Swinney, in Lévy Flights and Related Topics in Physics, M.F. Shlesinger, G.M. Zaslavsky, and U. Frisch, eds. (Springer, Berlin, 1995).

[4] M. F. Shlesinger, G. M. Zaslavsky, and J. Klafter, Nature 363, 31 (1993).

[5] E. Bouchaud and M. Daoud, J. Phys. A 20, 1463 (1987). 
[6] G. M. Viswanathan, V. Afanasyev, S. V. Buldyrev, E. J. Murphy, P. A. Prince, and H. E. Stanley, Nature (London) 381, 413 (1996).

[7] C. K. Peng, S. V. Buldyrev, A. L. Goldberger, S. Havlin, F. Sciortino, M. Simons, and H. E. Stanley, Nature (London) 356, 168 (1992).

[8] P. Lévy, Théorie de l'addition des variables aléatoires (Gauthier-Villars, Paris, 1937).

[9] H. B. Callen, Thermodynamics and an Introduction to Thermostatistics (Wiley, New York, 1985).

[10] C. Tsallis, Physica A 221, 277 (1995).

[11] E. M. F. Curado and C. Tsallis, J. Phys. A 24, L69 (1991).

[12] M. H. Ernst, Phys. Rep. 78, 1 (1981).

[13] C. Tsallis, R. S. Mendes and A. R. Plastino, Physica A 261, 534 (1998).

[14] D. H. Zanette and P. A. Alemany, Phys. Rev. Lett. 75, 366 (1995).

[15] C. Beck, Phys. Rev. Lett. 87, 180601 (2001).

[16] S. Abe and A. K. Rajagopal, Europhys. Lett. 55 (2001) 6.

[17] Our notation is compatible with the "renormalized" Tsallis's formalism [13] and, therefore, differs from Abe and Rajagopal's. 